competency-based measurement. The Accreditation Council for Graduate Medical Education (ACGME), the governing body for this process in the United States, is transitioning to a system of collecting annual program performance and attainment of competencies by trainees as key measures of training program performance. This presentation will describe this new accreditation system and introduce, with examples, the concept of achieving "milestones" based on six core competencies. These competencies include: patient care, medical knowledge, practice-based learning and improvement, interpersonal/communication skills, professionalism, and systemsbased practice. This new approach recognizes that process measures and relying on clinical exposure alone may not be adequate in assuring that trainees have acquired the skills to practice independently in their field.

\section{CONTROVERSIES IN BREAST MILK FORTIFICATION}

doi:10.1136/archdischild-2012-302724.0118

G Zachariassen. HC Andersen Childrens' Hospital, Odense, Denmark

Inadequate nutrition has been associated with impaired brain development among preterm infants. Especially low protein intake has been proven to be the primary limiting factor responsible for growth failure among preterm infants during the first critical week(s) after birth. In order to recover or catch-up after these first weeks, feeding has to replace both the deficit accumulated and to meet the requirements for normal growth.

Human milk (HM) has many advantages in the nutrition of preterm infants. Protein-concentration in HM is though variable and decreases with the duration of breastfeeding.

Fortification of HM with a multicomponent fortifier makes it possible to increase the concentration of nutrients - especially protein to meet the nutritional requirements of the preterm infants.

Fortification of HM can be achieved by standard fortification or individualized fortification. With individualized target fortification, the fortification method is based on HM analyses, adding protein to reach the targeted intake.

Fortified HM is easily fed as long as the preterm infant has a nasogastric tube during hospitalization. After hospital discharge fortification of HM becomes a challenge, but can be established using a bottle with fortified HM as supplementation while breastfeeding

Among "healthy" preterm infants catch-up growth can be achieved before discharge, while "sick" and small for gestational age preterm infants might not achieve catch-up growth until months or years after discharge. A gradual return to normal for all growth variables while avoiding excessive weight gain should be the goal for nutrition of very preterm infants during and after hospital discharge.

\section{CHALLENGES IN PEDIATRIC TRIAGE AT THE EMERGENCY DEPARTMENT}

doi:10.1136/archdischild-2012-302724.0119

HA Moll. Erasmus MC - Sophia Children's Hospital, Rotterdam, The Netherlands

Triage aims to identify patients in the emergency department who need immediate care and those who can safely wait in order to decrease mortality and morbidity. The Manchester Triage system (MTS) is based on expert opinion. It is based on flowcharts and specific decision rules which allocate urgency level (1 to 5) for a broad population visiting the emergency department. The reliability is dependent of the clinical experience and MTS training of the triagist, unambiguous of the system and diversity in flowcharts and discriminators to match the patients. The fundamental problem in studies to validate triage tools is the lack of consensus of the reference standard for "true urgency". Does the MTS urgency predicts true urgency? A proxy reference standard can be a combination of vital signs, disease severity, resource use and follow-up or just hospitalization. The validity of the MTS in a large prospective study of 17600 children in the Netherlands was moderate and low for febrile children compared to the combined reference standard. Age related fever modifications were validated in a new sample and improved specificity while sensitivity remained similar. The modified MTS agreed in 37\% with the reference standard of urgency and 36\% were overtriage and $13 \%$ undertriage ( $2 \%$ by $>1$ category). Over and undertriage need to be balanced to have a safe triage system. Performance of the MTS in different settings and in large populations need to be studied. New modifications on flowchart or subgroup level might further improve the MTS.

\section{PROCEDURAL SEDATION AND ANALGESIA IN THE PAEDIATRIC EMERGENCY DEPARTMENT}

doi:10.1136/archdischild-2012-302724.0120

I Shavit. Rambam Medical Center, Haifa, Israel

Pain is one of the most common reasons for which children visit the emergency department (ED). Nevertheless, pain is frequently under-recognized and under-treated in this setting. Ongoing evidence continues to show the harmful effects of pain on children's behavior and development.

The treatment of acute pain and anxiety in children undergoing therapeutic and diagnostic procedures in the emergency department has improved dramatically over the last few years. The availability of noninvasive monitoring devices and the use of short acting sedative and analgesic medications enable physicians to conduct safe and effective sedation and analgesia treatment. In today's practice of paediatric emergency medicine, sedation and analgesia has been considered as the standard of care for procedural pain.

This lecture will be focused on the basic principles of paediatric procedural sedation and analgesia in the paediatric ED.

\section{SPECIAL ATTENTION FOR LONG-STAY PATIENTS?}

doi:10.1136/archdischild-2012-302724.0121

${ }^{1} \mathrm{C}$ van Leeuwen, ' $\mathrm{M}$ de Jong, ${ }^{1} \mathrm{~K}$ Kuiper, ${ }^{2} \mathrm{~J}$ Latour, ${ }^{1} \mathrm{M}$ van Dijk. 'Intensive Care; ${ }^{2}$ Neonatal Intensive Care, Erasmus MC - Sophia Children's Hospital, Rotterdam, The Netherlands

Background and aims PICU admission is a major event for children and their parents. Therefore our guidelines dictate that a primary care nurse is assigned to (expected) long-stay patients in any case after two weeks. The primary care nurse coordinates nursing care, informs and supports parents, and organizes multidisciplinary meetings. Because this guideline was not always followed we organized an awareness week. This study evaluates the effectiveness of this intervention.

Methods Data were collected retrospectively over two threemonth periods: before and after the awareness week. Background variables including the presence of a primary care nurse were collected for all patients admitted $>2$ weeks. The two periods were compared using chi square tests, Fisher exact tests and Mann-Whitney tests.

Results The percentage of patients assigned a primary care nurse dropped statistically significantly from $56.8 \%$ to $33.3 \%$, p=0.024). Length of stay was statistically significantly shorter in the after period. 